\title{
Scattering theory of interface resistance in magnetic multilayers
}

\author{
Gerrit E.W. Bauer, ${ }^{¥}$ Kees M. Schep, ${ }^{£}$ Ke Xia ${ }^{\not}$ and Paul J. Kelly ${ }^{\phi}$ \\ $¥$ Department of Applied Physics and DIMES, Delft University of Technology, 2628 CJ Delft, The Netherlands \\ ${ }^{£}$ Philips Research, Prof. Holstlaan 4, 5656 AA Eindhoven, The Netherlands and \\ $\phi_{\text {Faculty of Applied Physics and MESA+ Research Institute, }}$ \\ University of Twente, P.O. Box 217, 7500 AE Enschede, The Netherlands
}

(Dated: October 26, 2018)

\begin{abstract}
The scattering theory of transport has to be applied with care in a diffuse environment. Here we discuss how the scattering matrices of heterointerfaces can be used to compute interface resistances of dirty magnetic multilayers. First principles calculations of these interface resistances agree well with experiments in the CPP (current perpendicular to the interface plane) configuration.
\end{abstract}

\section{INTRODUCTION}

According to the scattering theory of transport developed by Landauer in 1957 [1], the conductance of a single-mode wave guide reads

$$
G^{L}=\frac{e^{2}}{h} \frac{T}{1-T},
$$

where $T$ is the probability for a (spinless) electron at the chemical potential which approaches the sample from a reservoir on the left to be transmitted to a reservoir on the right. $T=1$ represents perfect transmission and an infinite conductance. Much later, Büttiker [2] realized that this expression should be replaced with

$$
G^{L B}=\frac{e^{2}}{h} T .
$$

When $T \longrightarrow 1$, the conductance is now finite, which physically represents the geometrical Sharvin resistance of the device. For the general multi-channel situation with spin the two-terminal Landauer-Büttiker formula reads:

$$
G^{L B}=\frac{e^{2}}{h} \sum_{i \sigma j \sigma^{\prime}} T_{j \sigma^{\prime}, i \sigma}=\frac{e^{2}}{h} \sum_{i \sigma j \sigma^{\prime}}\left|t_{j \sigma^{\prime}, i \sigma}\right|^{2},
$$

where $t_{j \sigma^{\prime}, i \sigma}$ is the probability amplitude that an electron approaching the scattering region in transverse mode $i$ and with spin $\sigma$ will be transmitted into an outgoing state with transverse mode $j$ and spin $\sigma^{\prime}$ on the other side of the sample. Some of the rather subtle points of this topic are discussed in [3].

The Landauer-Büttiker formula was introduced into the field of magnetoelectronics in order to compute the (magneto)conductance of microstructured magnetic multilayers [3]. Asano et al. [4] evaluated the effect of disorder numerically, whereas Brataas and Bauer [5, 6] used perturbation theory specifically for the Current Perpendicular to the interface Plane (CPP) geometry [3, 7, 8, 9, 10]. The transmission coefficients are accessible to first-principles calculations, and $G^{L B}$ was computed for ballistic superlattices by Schep et al. [11], and for single, specular interfaces by Schep et al. [12], van Hoof et al. [13] and Stiles and Penn 14. Recent improvements make it possible to extend $a b$ initio studies to disordered interfaces 15 .

Schep et al. 12 pointed out that the point contact resistance $R^{L B}=1 / G^{L B}$ is not the appropriate quantity to compare with the interface resistances, $R^{I}$, which have been measured accurately by the Michigan State University collaboration for different systems [7. The experimental values are obtained by using the parameters of the series resistor model (in which interface resistances and bulk resistivities are simply added in series) to fit the measured data. The finding that the series resistor model works very well for the vast majority of samples is a strong indication for the absence of quantum size effects (see, however, the Discussion section), which can be rationalized by the disorder in metallic multilayers. In this "dirty" regime, transport can be described by semiclassical methods such as the Boltzmann equation which has been studied in detail for the CPP configuration by Valet and Fert [16]. However, these authors did not take into account the discontinuity of the electronic structure at interfaces [1] which is the main source of the interface resistance [12]. Schep et al. showed how the scattering matrix of an isolated interface should be incorporated into the Boltzmann equation and derived an explicit expression for the interface resistance in diffuse multilayers [12] which can be directly compared with experimental results or numerical solutions of the Boltzmann equation 17 . 
In the following we work out the details of the derivation of the interface resistance in a dirty environment, for which there was no room in the original article [12], discuss the results in the light of new developments, and compare them with experiments.

\section{SEMICLASSICAL TRANSMISSION}

Let us begin by disregarding unnecessary complications associated with spin. At low temperatures, the distribution function for the CPP configuration in the presence of a weak electric field normal to a layered system and at a specified plane in a layer with index $L$ can then be expanded as

$$
f_{L, i}^{ \pm}=f_{L, i}^{0}+\delta\left(\varepsilon_{L, i}-E_{F}\right)\left[\mu_{L}-E_{F}+\gamma_{L, i}^{ \pm}\right]
$$

where $i$ the state index, $E_{F}$ is the Fermi energy of the total system and the superscript \pm denotes whether the state $i$ is right or left moving. $f_{L, i}^{0}$ is the equilibrium (Fermi-Dirac) distribution function. $\mu_{L}-E_{F}$ is the local shift of the chemical potential, so $\mu_{L}-\mu_{L^{\prime}}$ is the potential drop between layers $L$ and $L^{\prime}$. $\gamma_{L, i}^{ \pm}$describes the anisotropic part of the non-equilibrium distribution function, which vanishes in the sum over states. Once the $\gamma_{L, i}^{ \pm}$are known, the conductance can be calculated as

$$
G_{\text {Total }}=\frac{e}{h} \sum_{i}\left[\gamma_{L, i}^{+}-\gamma_{L, i}^{-}\right] / \Delta \mu_{\text {Total }}
$$

where $\Delta \mu_{\text {Total }}$ is the potential drop over the total system. The spatially dependent distribution functions could be obtained from the general solutions of the linearized Boltzmann equation in the layers [16], determining the unknown linear coefficients by the boundary conditions at the interfaces. Here we will follow a different route by matching the distribution functions (4) at a given plane in each layer:

$$
\begin{aligned}
& f_{L^{\prime}, i}^{+}=\sum_{j \in L}\left(\mathbf{T}_{L L^{\prime}}\right)_{i j} f_{L, j}^{+}+\sum_{j \in L^{\prime}}\left(\mathbf{R}_{L L^{\prime}}^{\prime}\right)_{i j} f_{L^{\prime}, j}^{-} \\
& f_{L, i}^{-}=\sum_{j \in L}\left(\mathbf{R}_{L L^{\prime}}\right)_{i j} f_{L, j}^{+}+\sum_{j \in L^{\prime}}\left(\mathbf{T}_{L L^{\prime}}^{\prime}\right)_{i j} f_{L^{\prime}, j}^{-} .
\end{aligned}
$$

where the transmission $(\mathbf{T})$ and reflection $(\mathbf{R})$ probability matrices include bulk and interface scattering (see Fig. 1).

In the absence of an applied bias all distribution functions are the same which leads to the current conservation condition:

$$
1=\sum_{j \in L}\left(\mathbf{T}_{L L^{\prime}}\right)_{i j}+\sum_{j \in L^{\prime}}\left(\mathbf{R}_{L L^{\prime}}^{\prime}\right)_{i j}=\sum_{j \in L}\left(\mathbf{R}_{L L^{\prime}}\right)_{i j}+\sum_{j \in L^{\prime}}\left(\mathbf{T}_{L L^{\prime}}^{\prime}\right)_{i j}
$$

and

$$
\begin{aligned}
& \sum_{i \in L^{\prime}}\left[\sum_{j \in L}\left(\mathbf{T}_{L L^{\prime}}\right)_{i j}+\sum_{j \in L^{\prime}}\left(\mathbf{R}_{L L^{\prime}}^{\prime}\right)_{i j}\right]=N_{L^{\prime}} \\
& \sum_{i \in L}\left[\sum_{j \in L}\left(\mathbf{R}_{L L^{\prime}}\right)_{i j}+\sum_{j \in L^{\prime}}\left(\mathbf{T}_{L L^{\prime}}^{\prime}\right)_{i j}\right]=N_{L},
\end{aligned}
$$

where $N_{L}$ denotes the number of modes in layer $L$. Since the total transmittance does not depend on the sign of the applied bias:

$$
\begin{aligned}
\sum_{i \in L^{\prime}} \sum_{j \in L}\left(\mathbf{T}_{L L^{\prime}}\right)_{i j} & =\sum_{i \epsilon L} \sum_{j \in L^{\prime}}\left(\mathbf{T}_{L L^{\prime}}^{\prime}\right)_{i j} \\
\sum_{i, j \in L}\left(\mathbf{R}_{L L^{\prime}}\right)_{i j}-\sum_{i, j \in L^{\prime}}\left(\mathbf{R}_{L L^{\prime}}^{\prime}\right)_{i j} & =N_{L}-N_{L^{\prime}}
\end{aligned}
$$


FIG. 1:

Let us now locate $L$ and $L^{\prime}$ at equivalent positions in different unit cells of a superlattice [12]. The electronic structures are then identical and the scattering potential is symmetric, thus $\mathbf{R}=\mathbf{R}^{\prime}$ and $\mathbf{T}=\mathbf{T}^{\prime}$. Substituting Eq. (㺼) into (6, (6):

$$
\begin{aligned}
& \gamma_{L^{\prime}, i}^{+}=\left(\mu_{L}-\mu_{L^{\prime}}\right) \sum_{j}\left(\mathbf{T}_{L L^{\prime}}\right)_{i j}+\sum_{j}\left(\left(\mathbf{T}_{L L^{\prime}}\right)_{i j} \gamma_{L, j}^{+}+\left(\mathbf{R}_{L L^{\prime}}\right)_{i j} \gamma_{L^{\prime}, j}^{-}\right) \\
& \gamma_{L, i}^{-}=\left(\mu_{L^{\prime}}-\mu_{L}\right) \sum_{j}\left(\mathbf{T}_{L L^{\prime}}\right)_{i j}+\sum_{j}\left(\left(\mathbf{R}_{L L^{\prime}}\right)_{i j} \gamma_{L, j}^{+}+\left(\mathbf{T}_{L L^{\prime}}\right)_{i j} \gamma_{L^{\prime}, j}^{-}\right) .
\end{aligned}
$$

The superlattice symmetry implies:

$$
\gamma_{L, i}^{ \pm}=\gamma_{L^{\prime}, i}^{ \pm} \equiv \gamma_{i}^{ \pm} ; \Delta \mu \equiv \mu_{L}-\mu_{L^{\prime}}
$$

which leads to

$$
\sum_{j}\left[\delta_{i j}-(\mathbf{T})_{i j}+(\mathbf{R})_{i j}\right]\left(\gamma_{j}^{+}-\gamma_{j}^{-}\right)=2 \Delta \mu \sum_{j}(\mathbf{T})_{i j}
$$

We can now define a local conductance $G^{S}$ which can be written in a matrix notation as

$$
G^{S}=\frac{e}{h} \sum_{i} \frac{\gamma_{j}^{+}-\gamma_{j}^{-}}{\Delta \mu}=\frac{2 e^{2}}{h} \sum_{i j}\left[(\mathbf{I}-\mathbf{T}+\mathbf{R})^{-1} \mathbf{T}\right]_{i j}
$$

Note that the factor 2 is not related to spin. In the single mode limit this corresponds to Landauer's "old" formula (1), but differs from the many-channel generalization derived by Büttiker et al. [18].

The transmission and reflection probability matrices of the superlattice unit cell in (17) can be constructed via concatenation of the scattering probabilities of the individual constituents, i.e. interfaces and bulk materials. The transmission $\mathbf{T}_{12}, \mathbf{T}_{12}^{\prime}$ and reflection $\mathbf{R}_{12}, \mathbf{R}_{12}^{\prime}$ of a system of two scatterers (subscript 1 and 2) reads 6 , 19, 20:

$$
\begin{aligned}
\mathbf{T}_{12} & =\mathbf{T}_{2}\left(\mathbf{I}-\mathbf{R}_{1}^{\prime} \mathbf{R}_{2}\right)^{-1} \mathbf{T}_{1} \\
\mathbf{R}_{12} & =\mathbf{R}_{1}+\mathbf{T}_{1}^{\prime} \mathbf{R}_{2}\left(\mathbf{I}-\mathbf{R}_{1}^{\prime} \mathbf{R}_{2}\right)^{-1} \mathbf{T}_{1} \\
\mathbf{T}_{12}^{\prime} & =\mathbf{T}_{1}^{\prime}\left(\mathbf{I}-\mathbf{R}_{2} \mathbf{R}_{1}^{\prime}\right)^{-1} \mathbf{T}_{2}^{\prime} \\
\mathbf{R}_{12}^{\prime} & =\mathbf{R}_{2}^{\prime}+\mathbf{T}_{2} \mathbf{R}_{1}^{\prime}\left(\mathbf{I}-\mathbf{R}_{2} \mathbf{R}_{1}^{\prime}\right)^{-1} \mathbf{T}_{2}^{\prime}
\end{aligned}
$$


The semiclassical concatenation of matrices implies that scattered electrons lose phase memory when returning to the point of departure. As mentioned above, there is much evidence that magnetoelectronic devices to date are very dirty. Scattering at bulk layers and/or interfaces is therefore diffuse, which in three dimensional systems suppresses interference. Indeed it can be proven by Random Matrix Theory that transport is governed by the above concatenation rules [21]. This proof requires "isotropy" and is valid to first order in an expansion of $1 / N$, where $N$ is the number of conduction channels [21]. In transition metal systems this should be an excellent approximation, with the exception of few-atom point contacts or break junctions.

The isotropy assumption of scattering means that the elements of the scattering matrix are equivalent and can be replaced by a constant value. If all elements of two matrices $\mathbf{X}$ (square, dimension $N \times N)$ and $\mathbf{Y}($ dimension $N \times M$ ) do not depend on their indices it is easy to show that

$$
\sum_{i j}\left([\mathbf{I}-\mathbf{X}]^{-1} \mathbf{Y}\right)_{i j}=\frac{\sum_{i j}(\mathbf{Y})_{i j}}{1-\frac{1}{N} \sum_{i j}(\mathbf{X})_{i j}}
$$

\section{DIFFUSE TRANSPORT}

If the transmission though a bulk layer (material $\alpha$ ) is ballistic we simply have $\left(\mathbf{T}_{\alpha}\right)_{i j}=\delta_{i j},\left(\mathbf{R}_{\alpha}\right)_{i j}=0$. In the presence of diffuse scattering, the following choice parameterizes the material and layer thickness dependence:

$$
\left(\mathbf{T}_{\alpha}\right)_{i j}=\left(\mathbf{T}_{\alpha}\right)_{i j}^{\prime}=\frac{1}{N_{\alpha}} \frac{1}{1+s_{\alpha}} ;\left(\mathbf{R}_{\alpha}\right)_{i j}=\left(\mathbf{R}_{\alpha}\right)_{i j}^{\prime}=\frac{1}{N_{\alpha}} \frac{s_{\alpha}}{1+s_{\alpha}}
$$

where

$$
s=\frac{e^{2}}{h} \frac{\rho d N}{A}
$$

consists of the bulk resistivity $\rho$, layer thickness $d$, and cross-section $A$. If we straightforwardly apply Eq. (3), we obtain for the dimensionless "point contact" conductance:

$$
g_{\alpha}^{L B} \equiv \frac{h}{e^{2}} G_{\alpha}^{L B}=\sum_{i j}\left(\mathbf{T}_{\alpha}\right)_{i j}=\frac{1}{\frac{1}{N_{\alpha}}+\frac{e^{2}}{h} \frac{\rho_{\alpha} d_{\alpha}}{A}}
$$

where we recognize the (dimensionless) Sharvin resistance $r_{\alpha}^{S h}=1 / N_{\alpha}$ in series with the (dimensionless) conventional bulk resistance

$$
r_{\alpha}=\frac{e^{2}}{h} \frac{\rho_{\alpha} d_{\alpha}}{A}
$$

Substituting the expressions (23) into Eq. (17), the conductance can be evaluated using Eq. (22) to yield the expected result:

$$
g_{\alpha}^{S}=2 \sum_{i j}\left[\left(\mathbf{I}-\mathbf{T}_{\alpha}+\mathbf{R}_{\alpha}\right)^{-1} \mathbf{T}_{\alpha}\right]_{i j}=\frac{1}{r_{\alpha}}
$$

The transmission through an interface $(I)$-bulk $(\alpha)$ material is:

$$
\mathbf{T}_{I \alpha}=\mathbf{T}_{\alpha}\left(\mathbf{I}-\mathbf{R}_{I}^{\prime} \mathbf{R}_{\alpha}\right)^{-1} \mathbf{T}_{I}
$$

The matrix $\mathbf{R}_{\mathbf{I}}^{\prime} \mathbf{R}_{\boldsymbol{\alpha}}$ in the denominator stands for the multiple reflection of electrons between interface and bulk material. $\mathbf{T}_{\mathbf{I} \boldsymbol{\alpha}}$ can be worked out under the random matrix assumption and

$$
g_{I \alpha}^{L B}=\sum_{i j}\left(\mathbf{T}_{I \alpha}\right)_{i j}=\left[\frac{1}{g_{I}^{L B}}+\frac{e^{2}}{h} \frac{\rho_{\alpha} d_{\alpha}}{A}\right]^{-1},
$$

where now the Sharvin conductance $N_{\alpha}$ in (25) is replaced by the Landauer-Büttiker interface conductance:

$$
g_{I}^{L B}=\frac{1}{r_{I}^{L B}}=\sum_{i j}\left|t_{i j}^{I}\right|^{2}
$$


For a single interface $\alpha|I| \beta$ we need to evaluate

$$
\mathbf{T}_{\alpha I \beta}=\mathbf{T}_{I \beta}\left(I-\mathbf{R}_{\alpha} \mathbf{R}_{I \beta}\right)^{-1} \mathbf{T}_{\alpha}
$$

which proceeds along the same lines. The hetero point contact resistance is:

$$
r_{\alpha I \beta}^{L B}=\frac{1}{\sum_{i j}\left(\mathbf{T}_{\alpha I \beta}\right)_{i j}}=r_{\alpha}+r_{\beta}+r_{I}^{L B} .
$$

The trilayer $\alpha|I| 2 \beta|I| \alpha$ has the transmission

$$
\mathbf{T}_{\alpha I 2 \beta I \alpha}=\mathbf{T}_{\beta I \alpha}\left(I-\mathbf{R}_{\alpha I \beta}^{\prime} \mathbf{R}_{\beta I \alpha}\right)^{-1} \mathbf{T}_{\alpha I \beta}
$$

and resistance

$$
r_{\alpha I 2 \beta I \alpha}^{S}=2\left(r_{\beta}+r_{I}^{L B}+r_{\alpha}\right)-r_{\alpha}^{S h}-r_{\beta}^{S h}
$$

in contrast to the Landauer-Büttiker result:

$$
r_{\alpha I 2 \beta I \alpha}^{L B}=2\left(r_{\beta}+r_{I}^{L B}+r_{\alpha}\right)
$$

In the series resistor model [7],

$$
A R_{\alpha I 2 \beta I \alpha}^{T}=2\left(A R_{\beta}+A R_{I}+A R_{\alpha}\right)
$$

which agrees with the present equation if we identify:

$$
A R_{I} \equiv \frac{A h}{e^{2}}\left(r_{I}^{L B}-\frac{r_{\alpha}^{S h}+r_{\beta}^{S h}}{2}\right)=\frac{A h}{e^{2}}\left(\frac{1}{\sum_{i j}\left|t_{i j}^{I}\right|^{2}}-\frac{1}{2}\left[\frac{1}{N_{\alpha}}+\frac{1}{N_{\beta}}\right]\right),
$$

which is the basic equation derived in [12]. The interface resistance in a diffuse environment is therefore not equal to the Landauer-Büttiker point contact resistance, but it can be obtained easily from it by substracting the geometrical Sharvin resistance! This result is readily extended to the two-channel resistor model, which holds for superlattices with collinear magnetization and sufficiently weak spin-flip scattering. Note that in this formulation the spin accumulation does not play a role.

We can imagine a system in which bulk transmission is ballistic and interfaces are specular. Eq. (17) can then be calculated directly from the interface scattering matrix alone, two of which have to be concatenated in the case a single barrier [12]. That result would hold when the interfaces consist of specular islands much larger than the Fermi wave lengths, but thickness fluctuations of the layers which introduce the dephasing necessary for the semiclassical method [12, 21].

\section{DISCUSSION}

The method described here relies on several assumptions, the validity of which must be tested. From a theoretical point of view, it is satisfying that as far as the transmission probability matrix is concerned, our results agree with Random Matrix Theory [21]. Furthermore, it is important to note that the expression for the interface resistance (37) does not depend on the bulk layer thickness or resistivity, in agreement with experiment. The assumptions of the model can be tested directly by comparison with numerically exact calculations for finite disordered systems [15]. The parameter-free calculation of transport properties 15 are based on the surface Green's function method implemented with a tight-binding linear muffin tin orbital basis 22]. Because a minimal basis set is used, we are able to carry out calculations for large lateral supercells and model disorder very flexibly within these supercells without using any adjustable parameters. The electronic structure is determined self-consistently within the local spin density approximation. For disordered layers the potentials are calculated using the layer CPA approximation [22]. The interface roughness was chosen as a bilayer of $50 \% / 50 \%$ alloy, but the results are not sensitive to moderate variations of alloy thickness and concentration, like a $60 \% / 40 \% 40 \% / 60 \%$ alloy concentration profile or between one and two mixed interface layers.

Although the derivation of the interface resistance was based on the assumption of superlattice periodicity, this construction should only be viewed as a convenient model for a diffuse environment which is in fact less restrictive. 
In a diffuse medium, the periodic boundary condition imposed above on the $\gamma_{i}$ can be replaced by the equality of the state-averaged $\sum_{i} \gamma_{i}$ on both sides because the scattering matrix of a single interface sandwiched by diffuse bulk layers does not depend on the state index. It follows that in this limit Eq. (37) holds for a single interface as well.

The isotropy assumption underlying the series resistor model appears to be hold for most structures. It is still interesting and important to explore their limitations. Recently, theoretical evidence has been obtained that the resistor model can break down 10, 15, 17, 23, 24]. Deviations can occur in terms of a breakdown of the relaxation time approximation within the Boltzmann formalism [17], or in terms of quantum corrections [10, 15, 23, 24. These correction become significant for very clean or thin samples, but there is no consensus whether these have been observed in experiments on CPP spin valves [25, 26]. Other complications may be residual spin-flip scattering at interfaces, which we cannot yet treat by first principles.

Interface resistances are listed in Table 1 for a number of different systems. The differences between $A R_{I}^{S}$ and $A R_{I}^{L B}$ are very significant for highly transparent interfaces. The agreement of the computed interface resistances with experiments, which was already found to be good for specular interfaces of the $\mathrm{Co} / \mathrm{Cu}$ systems [12], is improved on including interface disorder [15]. Recently, CPP experiments on $\mathrm{Fe} / \mathrm{Cr}$ 27] showed that the spin-averaged resistance agrees very well with the theoretical prediction, but that the polarization dependence is not yet completely understood.

The model assumptions here differ from another semiclassical formalism, viz. the magnetoelectronic circuit theory [28]. There, the nodes in the circuit are taken to be at quasi-equilibrium $\left(g_{L, i}^{ \pm}=0\right)$, which is valid when the potentials drop exclusively over the resistive elements, which is the case for e.g. tunnel junctions, long wires, and point contacts, but not necessarily for magnetic multilayers. This implies, for example, that the angular magnetoresistance curves calculated by Huertas-Hernando et al. [29], should be modified along the lines expounded here [30 before a direct comparison with experiment [31] can be carried out.

\section{ACKNOWLEDGMENT}

We acknowledge discussions with Bill Pratt, Jack Bass, Arne Brataas, Yuli Nazarov, Daniel Huertas-Hernando, Yaroslav Tserkovnyak, and Maciej Zwierzycki as well as support by FOM and the NEDO joint research program (NAME).

[1] R. Landauer, IBM J. Res. Dev. 1, 223 (1957).

[2] M. Büttiker, IBM J. Res. Dev. 32, 317 (1988).

[3] M.A.M. Gijs and G.E.W. Bauer, Adv. Phys. 46, 285 (1997).

[4] A. Oguri, Y. Asano, and S. Maekawa, J. Phys. Soc. Japan 61, 2652 (1992); Y. Asano, A. Oguri, and S. Maekawa, Phys. Rev. B 48, 6192 (1993).

[5] G.E.W. Bauer, Phys. Rev. Lett. 69, 1676 (1992).

[6] A. Brataas and G.E.W. Bauer, Phys. Rev. B 49, 14684(1994).

[7] W.P. Pratt, Jr., S.-F. Lee, J.M. Slaughter, R. Loloee, P.A. Schroeder, and J. Bass, Phys. Rev. Lett. 66, 3060 (1991); J. Bass and W.P. Pratt, J. Magn. Magn. Mater. 200, 274 (1999).

[8] M.A.M. Gijs, S.K.J. Lenczowski, and J.B. Giesbers, Phys. Rev. Lett. 70, 3343 (1993).

[9] J.-P. Ansermet, J. Phys.-Cond Mat. 10, 6027 (1998).

[10] E.Y. Tsymbal and D.G. Pettifor, Solid State Phys. 56, 113 (2001).

[11] K.M. Schep, P.J. Kelly, and G.E.W. Bauer, Phys. Rev. Lett. 74, 586 (1995).

[12] K.M. Schep, J.B.A.N. van Hoof, P.J. Kelly, G.E.W. Bauer, and J.E. Inglesfield, Phys. Rev. B 56, 10805 (1997).

[13] J.B.A.N. van Hoof, K.M. Schep, A. Brataas, G.E.W. Bauer, and P.J. Kelly, Phys. Rev. B 59, 138 (1999).

[14] M. D. Stiles and D. R. Penn, Phys. Rev. B 61, 3200 (2000).

[15] K. Xia, P.J. Kelly, G.E.W. Bauer, I. Turek, J. Kudrnovský, and V. Drchal, Phys. Rev. B 63, 064407 (2001).

[16] T.Valet and A. Fert, Phys. Rev. B 48, 7099(1993).

[17] D. R. Penn and M. D. Stiles, Phys. Rev. B 59, 13338 (1999).

[18] M. Büttiker, Y. Imry, R. Landauer. and S. Pinhas, Phys. Rev. B 31, 6207 (1985).

[19] B. Shapiro, Phys. Rev. B 35, 8256 (1987).

[20] M. Cahay, M. McLennan, and S. Datta, Phys. Rev. B 37, 10125 (1988).

[21] X. Waintal, E.B. Myers, P.W. Brouwer, and D.C. Ralph, Phys. Rev. B 62, 12317 (2000).

[22] I. Turek et al., Electronic Structure of Disordered Alloys, Surfaces and Interfaces (Kluwer, Boston-London-Dordrecht, 1997); O.K. Andersen, O. Jepsen, and D. Glötzel, Highlights in Condensed Matter Theory, edited by F. Bassani, F. Fumi and M. P. Tosi, (North-Holland, Amsterdam, 1985), p. 59.

[23] E.Y. Tsymbal, Phys. Rev. B 62, R3608 (2000).

[24] A. Shpiro and P.M. Levy, Phys. Rev. B 63, 014419 (2001) 
[25] D. Bozec et al., Phys. Rev. Lett. 85, 1314 (2000).

[26] J. K. Eid et al. Phys. Rev. B 65, 054424 (2002).

[27] A. Zambano, K.Eid, R. Loloee, W.P. Pratt Jr., and J. Bass, J. Mag. Magn. Mat. Lett., in press.

[28] A. Brataas, Yu. V. Nazarov, and G.E.W. Bauer, Phys. Rev. Lett. 84, 2481 (2000); Eur. Phys. J. B 22, 99 (2001).

[29] D. Huertas-Hernando, G.E.W. Bauer, Yu.V. Nazarov, J. Mag. Mag. Mat., in press.

[30] G.E.W. Bauer, Y. Tserkovnyak, D. Huertas-Hernando, and A. Brataas, unpublished.

[31] L. Giacamoni, B. Dieny, W. Pratt, private communication.

Table I: Results of first principles calculation of Eq. (37) 15] and K. Xia et al., unpublished.

\begin{tabular}{cccccc}
\hline \hline system & roughness & $A R_{\text {maj }}^{S}\left(\mathrm{f} \Omega \mathrm{m}^{2}\right)$ & $A R_{\text {min }}^{S}\left(\mathrm{f} \Omega \mathrm{m}^{2}\right)$ & $A R_{\text {maj }}^{L B}\left(\mathrm{f} \Omega \mathrm{m}^{2}\right)$ & $A R_{\text {min }}^{L B}\left(\mathrm{f} \Omega \mathrm{m}^{2}\right)$ \\
\hline \hline $\mathrm{Au} / \mathrm{Ag}(111)$ & clean & 0.094 & 0.094 & 2.41 & 2.41 \\
\hline $\mathrm{Au} / \mathrm{Ag}(111)$ & 2 layers 50-50 alloy & 0.118 & 0.118 & 2.43 & 2.43 \\
\hline $\mathrm{Au} / \mathrm{Ag}(111)$ & exp. [7] & $0.100 \pm 0.008$ & $0.100 \pm 0.008$ & & \\
\hline $\mathrm{Co} / \mathrm{Cu}(100)$ & clean & 0.33 & 1.79 & 2.27 & 3.11 \\
\hline $\mathrm{Co} h \mathrm{Cu}(111)$ & clean & 0.60 & 2.24 & 2.67 & 3.65 \\
\hline $\mathrm{Co} / \mathrm{Cu}(111)$ & clean & 0.39 & 1.46 & 2.39 & 2.80 \\
\hline $\mathrm{Co} / \mathrm{Cu}(111)$ & 2 layers 50-50 alloy & 0.41 & $1.82 \pm 0.03$ & 2.40 & 3.14 \\
\hline $\mathrm{Co} / \mathrm{Cu}(111)$ & exp. [7] & $0.26 \pm 0.06$ & $1.84 \pm 0.14$ & & \\
\hline $\mathrm{Fe} / \mathrm{Cr}(100)$ & clean & 2.82 & 0.50 & 3.51 & 1.45 \\
\hline $\mathrm{Fe} / \mathrm{Cr}(100)$ & 2 layers 50-50 alloy & 0.99 & 0.50 & 1.68 & 1.45 \\
\hline $\mathrm{Fe} / \mathrm{Cr}(110)$ & clean & 2.74 & 1.05 & 4.22 & 3.17 \\
$\mathrm{Fe} / \mathrm{Cr}(110)$ & clean [14 & 2.11 & 0.81 & & 3.22 \\
$\mathrm{Fe} / \mathrm{Cr}(110)$ & 2 layers 50-50 alloy & 2.05 & 1.10 & 3.53 & \\
$\mathrm{Fe} / \mathrm{Cr}(110)$ & exp. [27] & $2.7 \pm 0.4$ & $0.5 \pm 0.2$ & & \\
\hline \hline
\end{tabular}




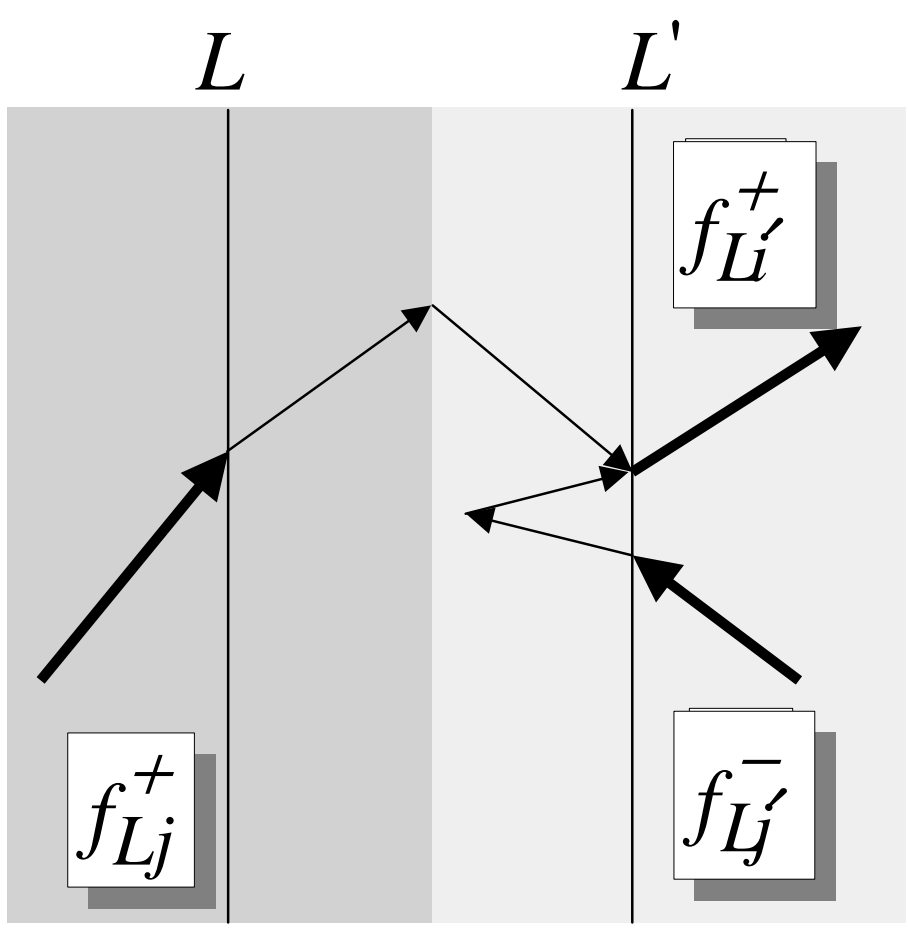

